\title{
Free classification: Element-level and subgroup-level similarity
}

\author{
STEPHEN HANDEL and JAMES W. RHODES \\ University of Tennessee, Knoxville, Tennessee 37916
}

\begin{abstract}
Subjects classified sets of multidimensional stimuli into two groups in any way they wished. The sets were composed of 6 or 12 stimuli: 2 or 4 instances of 3 different stimuli (e.g., 2 blue circles, 2 green circles, 2 red circles). There were striking individual differences in the preferred classification. Some subjects maximized the similarity between subgroups by matching the composition of the subgroupsone instance of each stimulus was placed in each group. The other subjects maximized the similarity among stimuli within each subgroup by placing similar stimuli in each group (the blues and greens in one group, the reds in the other). The nature of the stimuli as well as the relationships among the three stimuli had little effect on classification. In this case, cognitive styles specific to individuals but general across diverse dimensions and stimulus sets determined classification.
\end{abstract}

Imagine that you have two pennies, two nickels, and two dimes, and that your task is to place the coins in two groups in any way you wish.

There are many possibilities. Using color as the criterion dimension, you could place the nickels and dimes in one group, the pennies in the other; using monetary value, you could place the pennies and nickels in one group, the dimes in the other; or using size, you could place the pennies and dimes in one group, the nickels in the other. For each of these classifications, the motivation is to maximize the similarity within each subgroup by placing similar elements in the same group and to maximize the dissimilarity between subgroups by placing dissimilar elements in different groups.

An alternate approach to the classification task would be to place a penny, a nickel, and a dime in each group. Another solution would be to place two pennies and a nickel in one group, the two dimes and second nickel in the other group. Here the motivation is to maximize the similarity of the composition of each subgroup. The level of analysis is changed: the unit is the subgroup itself, not the elements within each subgroup. For the first possibility, the subgroups are matched-for each element in the first group, there is a matching element in the second. For the second possibility, the subgroups are balanced-. two of one kind and one of the third. This classification is more complex in that it attempts to balance the similarity of the elements within subgroups against the similarity among the subgroups. The similarity of

Preparation of this report was supported by the National Institute of Neurological and Communicative Disorders and Stroke, Grant NS 14842. Reprint requests should be sent to Stephen Handel, Department of Psychology, University of Tennessee, Knoxville, Tennessee 37916. the elements within each group is maximized since two of the three coins are kept intact in separate groups; the similarity of the subgroups is maximized since the groups are structurally identical.

Classifications that maximize the internal similarity of elements within each subgroup and classifications that maximize the external similarity between the subgroups themselves can be considered endpoints of a continuum. To the extent to which a classification maximizes element similarity, it will minimize subgroup similarity. Conversely, to the extent to which classifications maximize subgroup similarity, they will minimize the similarity among elements within each subgroup. (We will use the terms element-level and subgroup-level to label these two types of classifications.) Any classification, therefore, is a tradeoff between these extremes. Nonetheless, it is important to note that both are based on similarity and that similarity may be used for organization at all hierarchical levels.

To maximize the percentage of subgroup-level classifications, stimulus sets containing identical pairs (or triplets) of stimuli should be used so that one of each stimulus could be placed in each group. For pairs of stimuli, there would be two groups; for triplets there would be three groups. Unfortunately, few studies have used stimulus sets of this sort, since the goal of previous work was to investigate the basis by which subjects perceived element similarity. In one directly relevant experiment, Imai (1966) presented 12 colored Plexiglas chips made up of four chips of each of three colors and required subjects to place them into two groups. The majority of classifications $(53 \%)$ divided one color to form two balanced groups; each group contained six chips-four of one color, two of the divided color.

This outcome is suggestive in demonstrating the 
availability of classifications that maximize the similarity among subgroups. Unfortunately, it does not tell us much about the factors that contribute to their use. A goal of cognitive psychology may be said to describe the principles of organization that persons find useful and, if possible, to determine where each principle may be said to reside. In the present case, does the choice of classification depend on the type of stimulus variation-the number of stimuli, the similarity among stimuli, the type of dimensional structure, and so on? Or, are there "cognitive styles" specific to individuals that determine classification across diverse dimensions and stimulus sets? To investigate these questions, the composition of stimulus sets was varied to study the effect of stimulus factors while each subject classified every set to study the existence of individual differences in cognitive style or preference.

The basic stimulus set consisted of three different stimuli (e.g., blue circle, green circle, yellow square). Variations of these sets were presented to subjects. The variations chosen were based on outcomes from previous research. These included:

(1) Numerical properties. In half the sets there were 6 stimulus elements ( 2 of each stimulus); in the remaining half there were 12 stimulus elements (4 of each). Previous work has shown that subjects strongly prefer groups with equal numbers of elements and that subjects will choose a nonoptimal element-level classification to achieve numerical balance (Handel \& Imai, 1972; Imai, 1966). This pervasive tendencytoward numerical balance can be conceptualized as an attempt to achieve one type of subgroup-level similarity. If subjects maximize element-level similarity by placing the most similar pair of stimuli in one group, this would result in a $4 / 2$ split for 6 elements and an $8 / 4$ split for 12 elements. The numerical imbalance would be greater for sets of 12 stimulus elements. In contrast, subgroup-level classifications would result in numerical balance for both 6 and 12 elements. If numerical factors affect the choice of classification, then the percentage of element-level classifications should decrease when comparing sets of 6 elements with sets of 12 elements.

(2) Set structure. Sets were constructed such that the three stimuli differed along (a) one dimension (blue circle, green circle, yellow circle), (b) two dimensions in contingent fashion such that each pair of stimuli differed on both dimensions (yellow circle, blue square, green triangle), or (c) two dimensions such that two pairs differed on one dimension and one pair differed on two dimensions (blue circle, blue square, yellow square). For one-dimensional or contingent-dimensional sets, the only basis for element-level classification among the three stimuli would be the similarity between pairs of stimuli. Sub- jects could maximize element similarity by grouping together the two or four instances of the two most similar stimuli. However, for contingent-dimensional sets, each pair of stimuli would be more dissimilar, thereby reducing the achievable element-level similarity. In contrast, for dimensional sets, element-level classifications may be based on element similarity or on the dimensional structure (pairing yellow squares with blue squares or pairing blue circles with blue squares).

(3) Stimulus structure. When stimuli are constructed from levels of two or more dimensions, the dimensions may remain separable and easily analyzed or the dimensions may fuse to form a complex stimulus in which the component dimensions are not easily perceived (see Garner, 1974, for a review of the distinction between separable and integral dimensions). For separable stimuli, the basis for element-level classification would be dimensional structure (one group would be formed for each level of one dimension). ${ }^{1}$ For integral stimuli, the basis for element-level classification would be the perceived similarity between each pair of stimuli (Handel \& Imai, 1972). It can be hypothesized that for separable stimuli, element-level classification would be maximum for dimensional sets in which dimensional classification is possible, but that for integral stimuli, elementlevel classification should be maximum for sets in which two stimuli are highly similar.

In the present experiment, these three stimulus factors-number of stimulus elements, set structure, stimulus structure-were varied orthogonally to assess their effects separately and in combination. The goal of the analyses will be to measure the strength of these factors as opposed to individual differences in determining the type of grouping organization chosen.

\section{METHOD}

\section{Subjects}

The 28 subjects were all University of Tennessee undergraduates recruited from the introductory psychology class. All had normal color vision.

\section{Stimulus Dimensions}

Four different stimulus types were constructed from pairs of dimensions. Each dimension had four levels so that 16 different stimuli were possible for each type.

The dimensions of the first type were shape and color. The shapes were circle, square, triangle, and hexagon; the colors were blue, green, yellow, and red. The levels of each dimension were nominal and the discriminability among levels was high. The stimuli were made from colored construction paper. All forms were of equal subjective size (average area was $4.5 \mathrm{~cm}^{2}$ ) and were mounted on white matte cardboard backing measuring $5 \times 9 \mathrm{~cm}$.

The dimensions of the second set of stimuli were size of square and achromatic Munsell lightness. The discriminability among levels of each dimension was low. The levels of the size dimension in terms of the length of one side were $1.5,1.9,2.3$, and $2.7 \mathrm{~cm}$. The levels of the lightness dimension were N3, N4, N5, and N6. The squares were cut from glossy Munsell paper but not mounted. 
The dimensions of the third set of stimuli were also size of square and achromatic Munsell lightness. In contrast to the second set, the levels of each dimension were chosen to be highly discriminable. The levels of the size dimension in terms of the length of one side were $.3,1.5,2.7$, and $4.0 \mathrm{~cm}$. The levels of the lightness dimension were $\mathrm{N} 1, \mathrm{~N} 3.5, \mathrm{~N} 6$, and N8.5.

The dimensions of the fourth set of stimuli were Munsell value and chroma: all chips were the same hue-5RP. The levels of the value dimension were $/ 3 /, / 4 /, / 5 /$, and $/ 6 /$. The levels of the chroma dimension were $4,6,8$, and 10 . The chips were glossy $(2.5 \times 3.7 \mathrm{~cm})$ and mounted on white matte cardboard backing $(5 \times 9 \mathrm{~cm})$.

These four types were identical to those used previously (Handel \& Imai, 1972). A schematic for each set as well as the judged similarities between each pair of stimuli is given in that paper.

Previous work (Handel \& Imai, 1972) has suggested that the geometric shape $\times$ color stimuli and the size $\times$ lightness stimuli are perceived in terms of the dimensional structure; they are analyzable, or separable, stimuli. In contrast, the Munsell value $\times$ chroma stimuli are perceived in terms of the similarity among stimuli; they are unanalyzable, or integral, stimuli.

\section{Stimulus Sets}

Each set consisted of three different stimuli. In half the sets, there were 6 elements, 2 of each stimulus. In the other half, there were 12 elements, 4 of each stimulus.

There were three different kinds of stimulus sets. For onedimensional sets, each stimulus differed by one level along a single dimension (e.g., N3, N4, N5). Two sets were constructed for each of the eight dimensions coming from the four two-dimensional stimuli: one contained 6 elements ( 2 per stimulus), and one contained 12 elements ( 4 per stimulus). For contingent-dimensional sets, each pair of stimuli differed on two dimensions (e.g., blue square, green triangle, yellow hexagon). There were 12 sets in all: 4 for the geometric shape $x$ color dimensions, 2 for the low-discriminability size $x$ lightness dimensions, 2 for the high-discriminability size $\times$ lightness dimensions, and 4 for the Munsell value $\times$ chroma dimensions. As above, half the sets contained 6 elements and half contained 12 elements. For dimensional sets, one pair of stimuli differed on a single dimension, a second pair differed on the other dimension, and the third pair differed on both dimensions (e.g., blue square, yellow square, yellow triangle). There were 8 sets in all: 2 for each of the four dimension-pairs, 1 set containing 6 elements and 1 containing 12 elements.

Representative sets are shown schematically in Figure 1. Each set may be represented in a matrix, with the rows and columns representing the levels of each dimension. For one-dimensional sets, the stimuli are portrayed in a column matrix; for contingentdimensional and dimensional sets, the stimuli are portrayed in a $3 \times 3$ matrix. Sets of 6 stimuli are pictured: the 2 elements of each stimulus are shown in each appropriate cell (for sets of 12 stimuli, there would have been 4 elements in each cell). The experimental sets sampled among all the 16 possible stimuli so that each stimulus was used equally often.

\section{Procedure}

The experimenter faced the subject across the table and placed the stimuli in front of the subject in a random arrangement. The subjects were free to place the stimuli into any number of groups in any way they wished. Following this, the subjects were required to classify into two groups in any way they wished.

The order of presentation of the sets was counterbalanced across subjects. All sets were presented on one session of about $40 \mathrm{~min}$.

\section{RESULTS}

The vast majority of free classifications were into three groups with each stimulus defining one subgroup. Over $75 \%$ of the classifications were this type, and there were no differences among stimulus sets or subjects.

For two-group classification, preliminary analyses indicated that there were large individual differences in the preferred classification. For this reason, the first step was to create a subject by subject overlap matrix and cluster subjects with identical two-group response patterns. Four clusters emerged. ${ }^{2}$ These four, characterized by the predominant type of classification, are listed below:

(1) Matched subgroup-level classification. Matched classifications were those in which the two subgroups were identical. With sets of 6 elements, each group of 3 contained 1 of each stimulus; with sets of 12 elements, each group of 6 contained 2 of each stimulus. Eight subjects followed this strategy; $93 \%$ of their classifications were this type.

(2) Element-level classification. Element classifications were those in which similar stimuli were placed in the same group. With sets of 6 elements, the two instances of the most similar pair of stimuli (i.e., adjacent) would be placed in one group. There are two possible element classifications for each set. For instance, the element-level classifications for each set N3, N3, N4, N4, N5, N5 would be N3, N3, N4, N4// $\mathrm{N} 5, \mathrm{~N} 5$ or N3, N3//N4, N4, N5, N5. For sets of 12 elements, the four instances of the 2 most similar stimuli would be placed in one group. Eight subjects utilized this strategy: $85 \%$ of their classifications were this type.

(3) Matched subgroup-level and element-level classifications. Five subjects alternated between these two types of classification, although matched classifications predominated: $66 \%$ were matched subgrouplevel classifications and $25 \%$ were element-level classifications.

(4) Matched and balanced subgroup-level classifications. Balanced subgroup-level classifications are those in which the elements of one stimulus are split and half the elements are grouped with each of the other stimuli. For example, the classification four red circles, two green circles//two green circles, four yellow circles is a balanced classification. For each set, there are three possible balanced classifications, achievable by splitting one of the three stimuli. Six subjects used both types of subgroup-level classifications: $64 \%$ were matched and $17 \%$ were balanced. No subject used balanced subgroup-level classifications as his preferred mode.

These results are summarized in Figure 1. For each cluster of subjects representing a response strategy, the percentages of element-level, matched subgrouplevel, and balanced subgroup-level classifications are shown separately for one-dimensional, contingentdimensional, and dimensional sets. The results for sets of 6 and 12 stimuli were averaged, since there were no differences due to the number of stimuli.

The major effect due to stimulus variables was the 


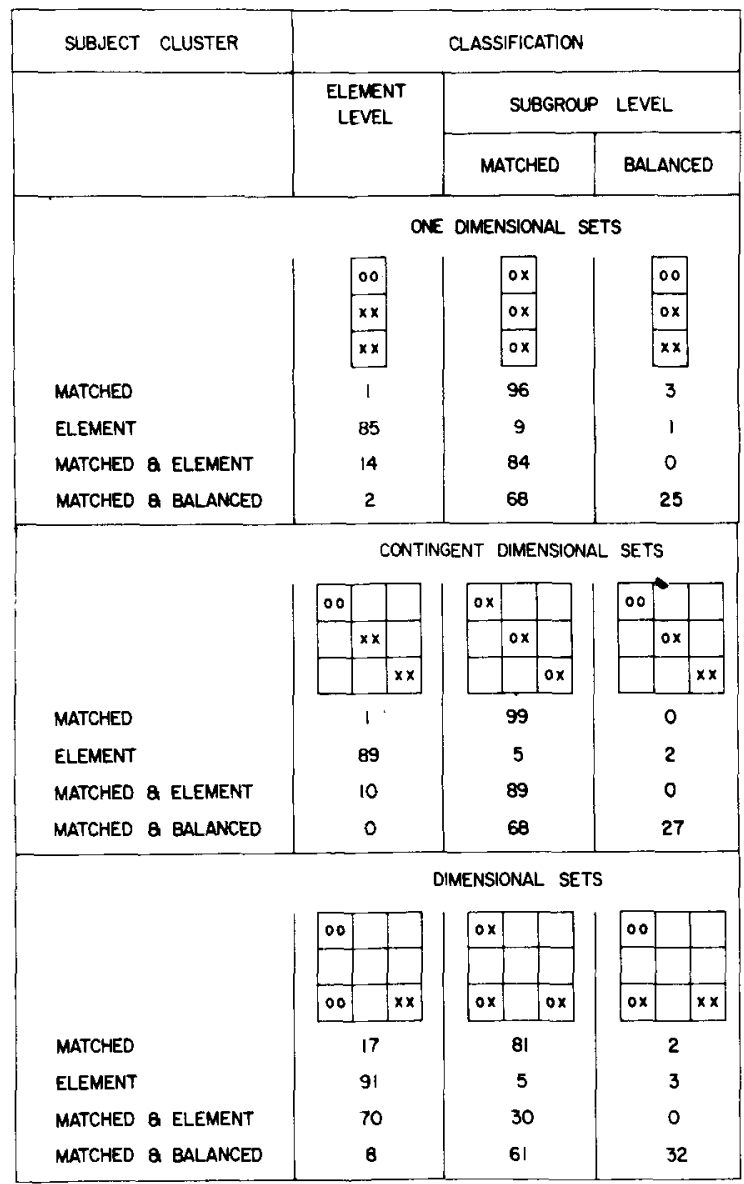

Figure 1. The percentage of element-level, matched subgrouplevel, and balanced subgroup-level classifications for each cluster of subjects. One-dimensional, contingent-dimensional, and dimensional sets are illustrated separately. Each set contains six stimuli, and the three different stimuli (two examples of each) are shown in the appropriate cells for each type of set. For elementlevel, matched subgroup-level, and balanced subgroup-level classifications, the elements comprising one group are represented by " $O$ ", and the elements in the second group are represented by " $x$ ".

interaction between type of stimulus and type of set. Overall, element-level classifications were nearly twice as frequent for dimensional as for either one- or contingent-dimensional sets $(47 \%$ to $28 \%)$. However, this increase was not consistent across all types of stimuli; it was due mainly to the increased use of element-level classification for separable dimensions. Consider those subjects who utilized both matched subgroup-level and element-level classifications. For separable dimensions (e.g., color $X$ shape, size $\times$ lightness), element-level classification was highly preferred $(83 \%)$ ). In contrast, for integral dimensions (e.g., Munsell value $\times$ chroma), matched subgrouplevel classification was highly preferred $(69 \%)$. This same effect was found in weaker form for the other classification strategies; for example, for those subjects preferring matched subgroup-level classification, all element-level classifications (17\%) occurred for separable dimensions.

\section{DISCUSSION}

The results are clear-cut: Differences in classification were due to individual strategies. No overall preferences were found between the two types of classification. Roughly equal numbers of subjects preferred element-level and subgroup-level classification, although matched subgroup-level classification was preferred to the more complex balanced subgrouplevel classification. The effects of stimulus variables were small and primarily influenced the classification of subjects who alternated among the possible classifications.

It is important to note that the nature of the stimulus dimensions did not directly affect classification. While the separable-integral distinction can be utilized to predict the nature of element-level classifications (Handel \& Imai, 1972), it does not predict the choice between element-level and subgroup-level classifications. The only exception to this contention was for dimensional sets in which element-level classifications increased for separable dimensions but not for integral dimensions. This makes good sense, since the dimensional structure would not be easily perceivable for the integral Munsell value $\times$ chroma stimuli and so would not act as an impetus for element-level classification.

In general, the choice of classification should depend jointly on the nature of the stimuli, the nature of the response, and individual preferences. In the present experiment, it is true that only a small number of the possible sets or stimuli have been sampled. Nevertheless, we would argue that the choice between element-level and subgroup-level classification would be relatively insensitive to these sorts of variations in a free-classification task. The decision to use an element-level or subgroup-level classification seems essentially a strategic one. This is not to say that classification cannot be dependent on the perceptual characteristics of the stimuli or on the response requirements of the task. Rather, it implies that the two classifications are alternate realizations of the perceptual structure and that here the choice between the two is not closely determined by the properties of that structure or by the nature of the responses.

This outcome is not true in other contexts. For example, Handel and Todd (in press) asked subjects to segment the elements of a repeating sequential pattern into subgroups to produce a pattern that seemed better and more rhythmical than other possible groupings. In this case, the type of response-whether the response was made vocally, or by tapping out the pattern on bongo-drums, or by drawing slash marks on a spatial-visual array-appeared to determine the 
availability of element-level as opposed to subgrouplevel groupings. However, within the constraint imposed by the response requirements, the choice of grouping was based purely on individual preferences.

In sum, then, each context yields a unique configuration of stimulus, response, and individual determinants. We need to characterize the properties of the context (psychological and even possibly sociological) that determine the classification or grouping (see Simon, 1972, for a similar viewpoint).

\section{REFERENCES}

GaRner, W. R. The processing of information and structure. Potomac, Md: Erlbaum, 1974.

HANDEL, S., \& ImAi, S. The free classification of analyzable and unanalyzable stimuli. Perception \& Psychophysics, 1972, 12, 108-116.

HANDEL, S., \& TODD, P. The segmentation of sequential patterns. Journal of Experimental Psychology: Human Perception and Performance, in press.
ImaI, S. Classification of sets of stimuli with different stimulus characteristics and numerical properties. Perception \& Psychophysics, 1966, 1, 48-54.

Simon, H. A. Complexity and the representation of patterned sequences of symbols. Psychological Review, 1972, 79, 369-382.

\section{NOTE}

1. It may be that dimensional classification also maximizes the element similarity within a subgroup, but this need not be the case. Consider the set yellow circle, yellow square, blue square. The yellow circle and yellow square may be grouped together either because they are more similar than are the yellow square and blue square or because the subject prefers to classify on the basis of color, without regard to similarity at all. Nonetheless, the goal is to contrast the subgroups by placing dissimilar stimuli in each group, and thus both will be considered element-level classifications.

2 . The four clusters accounted for 27 of the 28 subjects. The data from the remaining subject were not included in the analysis.

(Received for publication January 16, 1980; revision accepted June 4,1980 .) 